\title{
Potential Biomarkers of Schizophrenia from MEG Resting-State Functional Connectivity Networks: Preliminary Data
}

\author{
Susan M. Bowyer ${ }^{1,2 *}$, Klevest Gjini' ${ }^{3}$, Xiao Zhu1 ${ }^{1}$, Lawrence Kim¹, John E. Moran1, \\ Syeda U. Rizvi' ${ }^{1}$, Valentina Gumenyuk ${ }^{1}$, Norman Tepley ${ }^{1}$, Nash N. Boutros $^{4}$ \\ ${ }^{1}$ Department of Neurology, Henry Ford Hospital, Detroit, USA \\ ${ }^{2}$ Department of Neurology, Wayne State University, Detroit, USA \\ ${ }^{3}$ Department of Neurosurgery, Seton Brain and Spine Institute, Austin, USA \\ ${ }^{4}$ Department of Psychiatry, University of Missouri, Kansas City, USA \\ Email: sbowyer1@hfhs.org
}

Received 30 October 2014; revised 19 November 2014; accepted 3 December 2014

Academic Editor: Inga Poletaeva, Lomonosov Moscow State University, Russia

Copyright (C) 2015 by authors and Scientific Research Publishing Inc.

This work is licensed under the Creative Commons Attribution International License (CC BY). http://creativecommons.org/licenses/by/4.0/

(c) (i) Open Access

\section{Abstract}

Previous studies examining coherence and connectivity deviations in schizophrenia patients relied on standard coherence measures between recording sites (at the sensor level). A coherence source imaging (CSI) methodology where coherence is assessed within imaged brain structures (at the source level) was developed recently by our group and applied successfully for detecting coherent areas in the cortical networks of patients with epilepsy. We applied this Magnetoencephalography (MEG)-CSI technique to measure normal and pathological patterns of brain oscillations (biomarkers) in normal subjects and patients diagnosed with schizophrenia. Twelve patients diagnosed with schizophrenia and twelve healthy control subjects were studied. A ten-minute resting state MEG brain scan was performed with eyes open. MEG-CSI analysis was performed to identify the cortical areas that interacted strongly within the $3-50 \mathrm{~Hz}$ frequency range. Statistically significant increased regions of coherence were detected in schizophrenia patients compared to controls in the right inferior frontal gyrus (BA 47-pars orbitalis), left superior frontal gyrus (BA9dorsolateral prefrontal cortex), right middle frontal gyrus (BA 10-anterior prefrontal cortex \& BA 46-dorsolateral prefrontal cortex), and right cingulate gyrus (BA 24-ventral anterior cingulate cortex). These areas are involved in language, memory, decision making, empathy, executive and, higher cognitive functioning. We conclude that MEG-CSI can detect imaging biomarkers from resting state brain activity in schizophrenia patients that deviates from normal control subjects in several behaviorally salient brain regions. Analysis with MEG-CSI can provide biomarkers of ab-

\footnotetext{
Corresponding author.
}

How to cite this paper: Bowyer, S.M., Gjini, K., Zhu, X., Kim, L., Moran, J.E., Rizvi, S.U., Gumenyuk, V., Tepley, N. and Boutros, N.N. (2015) Potential Biomarkers of Schizophrenia from MEG Resting-State Functional Connectivity Networks: Preliminary Data. Journal of Behavioral and Brain Science, 5, 1-11. http://dx.doi.org/10.4236/jbbs.2015.51001 
normalities in the resting-state. The findings and procedures described can be used to probe the pathophysiology of schizophrenia and possibly detect subtypes.

\title{
Keywords
}

\author{
Magnetoencephalography (MEG), Coherence, Schizophrenia, Functional Resting State Brain \\ Networks
}

\section{Introduction}

Schizophrenia is a psychiatric disorder that causes a patient to have remarkably different perceptions of reality than what is apparent to the people around them. This disorder affects approximately $1 \%$ of the world's population (70 million) over the age of 18 [1]. It is primarily characterized by hallucinations (auditory, visual, tactile, gustatory, and olfactory), delusions, disordered thinking, irregular emotional expressions, and suicidal behavior [2]-[4]. The disorder is characterized by a wide range of symptoms including positive (non-deficit), negative (deficit) and cognitive disturbances [2]-[5].

The etiology and pathophysiology of schizophrenia remain elusive. A combination of genetic predisposition and environmental stress is hypothesized as the root of the illness. A number of neurotransmitters have been implicated from excess dopamine to reduced functioning of the N-methyl-D-aspartate glutamate (NMDA) receptor [1] [6]-[9]. There is currently no cure for schizophrenia, and the economic burden is high [10].

Non-invasive brain imaging studies have been performed with Electroencephalography (EEG), Magnetoencephalography (MEG), Positron Emission Tomography (PET) and functional Magnetic Resonance Imaging (fMRI) with the goal of identifying objective measures (imaging biomarkers) of the underlying pathological processes that can be used to diagnose this disorder. High electrode density (i.e., 64 or more channels) EEG examinations of the resting state brain activity in schizophrenia patients have revealed a generalized increase in slower EEG rhythms with a global decrease in EEG power mostly in the alpha and beta frequency ranges [11][13]. The reported topographical distribution of the abnormalities is not uniform with some reports emphasizing a frontal maximal deviation [11], and others pointing to the central and post-central/parietal areas [14]. To resolve the source localization of the EEG, we look to the fMRI results. The Default-Mode Network (DMN) detected by fMRI accounts for the persistent low frequency activity of the brain when it is not engaged in a focused task (i.e., resting-state) [15]. Several fMRI studies [16]-[20] of the resting state have shown coherence of low-frequency signal oscillations among brain regions supporting the theory that schizophrenia may be a network disorder. fMRI has found greater coherence in the DMN in the regions of the left frontal polar cortex, right dorsolateral prefrontal cortex, and multiple regions of the basal ganglia [21] in patient with schizophrenia. The irregular activation of the basal ganglia circuit was paired with the less active anterior cingulate cortex, suggesting a possible breakdown in the executive network function in patients with schizophrenia. A more recent fMRI has found cortico-subcortical disconnection within the frontal parietal network inpatients with schizophrenia [22]. This study also demonstrated that the schizophrenia patients had increased functional connectivity between several regions in the frontal parietal network and regions belonging to the primary sensory processing.

Synchronized activity, of both low and higher frequencies, within a neuronal network can be detected and localized using connectivity and coherence analysis of MEG data [23]. Magnetoencephalography (MEG) is a noninvasive technique for measuring magnetic fields emanating from the human brain. MEG can be a useful probe of the DMN and offers millisecond-scale temporal resolution [24]. The analysis of coherence between EEG electrode site [25] and MEG sensors [26] (at the sensor level) has been performed for many years. However, at best only regional inference of cortical connectivity can be estimated without first imaging brain activity (at the source level). MEG studies looking on the sensor level have investigated the resting state in schizophrenia patients and found a higher density of delta and theta generators primarily in temporal and parietal areas [27]. As a group, schizophrenia patients when compared with normal controls demonstrate significant decreases in alpha peak frequency and power [28]. In a group of stable medicated patients the dipole localization of both slow and fast activity dominated the temporoparietal region, in contrast to the central dipole distribution in control subjects [29]. Recently, techniques have been investigated that calculate the connectivity across the brain 
using coherence and phase synchrony measures between sources of MEG imaged brain activity in normal and abnormal patient populations [23] [30]-[32]. One such study used MEG imaged coherence between a voxel and the rest of the brain to detect difference is the resting state between patients with Schizophrenia and controls. They specifically looked at the eye closed condition and found impaired alpha coupling in frontal, parietal, and temporal regions [31]. Previously Rutter et al. [33] preformed a similar MEG study on this population again with the eyes closed and found patients with schizophrenia had significantly reduced activation in the gamma frequency band in the posterior region of the medial parietal cortex. These methods vary in the type of imaging technique applied to the MEG data, the number of sources for which paired coherence can be calculated, and the use of phase synchrony in place of coherence. These MEG studies have shown the possibility that this technique can provide important biomarkers for treatment.

Our recent study has combined our recursive current density imaging technique, MR-FOCUSS [34], with an Independent Component Analysis (ICA) technique that enhances the imaging of bursts of brain electric activity, such as epileptic spikes and other abnormal transients [35] [36] into a MEG coherent source imaging technique (MEG-CSI). Our recent paper demonstrated this MEG-CSI method technique is valuable in imaging the Epileptic network regions prior to surgical resection [36]. A similar coherent MEG imaging paradigm as the one used in this study has been used to image the temporal dynamics of power fluctuatons to assess the resting state brain networks in a group of normal control subjects [37]. We found similar results with our MEG-CSI method in our control subjects.

In this pilot study, we have illustrated the use of this MEG-CSI technique on data from patients with schizophrenia compared to controls subjects. We used MEG to examine the resting-state functional network in a group of stable medicated schizophrenia subjects compared to normal subjects. We expect that this type of MEGCSI analysis performed on the transient neuronal oscillations in specific regions of the brain, identified by past brain imaging studies in patients with schizophrenia, will provide biomarkers of abnormal functional connectivity indicative of this devastating disorder. Brodmann area nomenclature was used to identify the brain regions of interest. Detection of the magneto/electro-physiological abnormalities associated with schizophrenia will have important public health significance as a better understanding of the pathophysiological mechanisms in these patients will help in the development of early detection and monitoring responses to therapeutic interventions.

\section{Methods}

\subsection{Subjects}

Twelve patients who have been diagnosed with schizophrenia (mean age plus SD: $32 \pm 8.8$ years age; age range 19 - 45years; 10 males and 2 females) and 12 control subjects (mean age plus SD: $27 \pm 6.5$ years; age range 19 42 years; 4 males and 8 females) were studied with a 148 channel magnetometer whole head MEG system (Magnes WH2500, 4D-Neuroimaging). The inclusion criteria for the patient group were: 1) Male or female chronic schizophrenia outpatients meeting DSM-IV criteria for both schizophrenia and its subtype; 2) Negative drug screens upon admission to the study, in addition to a corroborated history of at least one month of an illicit drug-free period prior to admission; 3) Normal neurological examinations; 4) Age between 19 and 60; 5) All medications have been constant for at least four weeks at the time of recording. The exclusion criteria were: a) Patients with history of neurological disorders, including head trauma causing any period of loss of consciousness; b) Axis II diagnoses; c) Pregnancy; d) Active substance abuse (positive urine toxicity), or inability to verify abstinence with the exception of tobacco dependence.

The normal control subjects were recruited from our institution and an affiliated institution's employee population. This group included both men and women between 19 and 60 years old, matched for age (within \pm 5 years) with the subjects in the patient group. The exclusion criteria for the control group were: 1) History of psychiatric illnesses, as evidence by prior psychiatric hospitalizations, outpatient psychiatric treatment, or being on any psychotropic medication, except for a non-psychiatric reason, e.g., amitriptyline for migraine headache; 2) Current use of any medications, except occasional analgesics for headache; 3) History of neurological illnesses, including head trauma resulting in loss of consciousness; 4) History of psychiatric disorders in the immediate family; 5) History of substance abuse, or dependence; 6) Pregnant women (as evidenced by urine or blood tests). Smoking was allowed up to arriving in the laboratory which is about one hour before recording. Finally, any social alcohol consumption was restricted to more than 24 hours prior to testing.

All subjects and patients gave written informed consent prior to this study. The Institutional Review Boards at 
Henry Ford Health System and Wayne State University approved the protocol. All patients were recruited from the outpatient clinics of the Wayne State University Department of Psychiatry. Patients were enrolled as they were found to have met inclusion criteria and agreed to participate. As such these patients represent the general population of schizophrenia with predominantly positive symptoms. There were no patients enrolled with predominantly negative symptoms.

\subsection{MEG Data Collection Procedures}

Neuromagnetic recordings were carried out to measure spontaneous resting-state brain activity. Each subject removed all metal from his/her body and changed into a hospital gown. We taped 3 localization coils to the subject's forehead and 1 in front of each ear [38]. The subject then lay comfortably on bed, inside the magnetically shielded room. The subjects head shape was digitized along with the location of the 5 coils. The neuromagnetometer helmet containing the detector array was then placed around the subject's head in close proximity to most of the cortical surface. The subject was asked to avoid excessive eye blinks and body movements during data collection.

MEG data were sampled at a rate of $508 \mathrm{~Hz}$ with a low pass filter set to $0.1 \mathrm{~Hz}$ and the high pass filter set to $100 \mathrm{~Hz}$. Changes in the subject position between the beginning and end of a study were detected by changes in magnetic fields from the coils on the forehead and ears. Runs during which the subject's head shifted position more than $0.5 \mathrm{~cm}$ were repeated. Each subject was monitored by video camera and two-way audio speaker system during the time he/she was in the shielded room.

Spontaneous resting-state brain activity was recorded by MEG for 10 minutes, while the subject was lying quietly on his/her back, with eyes open. The subjects were told to keep their eyes open in order to eliminate coherent activity in the occipital cortex corresponding to alpha activity ( 8 - $13 \mathrm{~Hz}$ range). The patient's eyes were monitored during the 10 minute collection via a camera mounted in the room attached to a monitor. A standard MRI scan, rescaled to each patient's digitized head shape, was utilized to display the MEG results.

\subsection{MEG Data Analysis}

All data processing was performed with our software, MEG-Tools [39], using MATLAB. Data were forward and backward filtered to 3 - $50 \mathrm{~Hz}$. Independent Component analysis (ICA) was used to remove heart activity artifacts from the raw MEG data. Then singular value decomposition (SVD) of MEG data was used if needed to eliminate other noise components, such as dental artifact, not removed by the ICA and frequency filtering.

Coherence source imaging (CSI) analysis [36] was performed on the 10 minutes of resting-state MEG data to identify cortical sources that interacted strongly within each frequency. To localize cortical source activity, a head model was constructed using a standard T1-weighted high-resolution volumetric MR image. The source space consisted of X-, Y- and Z-oriented dipoles at approximately 4000 locations, distributed to represent the same amount of gray matter identified in the MR image. The MR images were co-registered with the individual's digitized head shape recorded at the time of MEG data collection.

The 10 minutes of resting-state MEG data were prepared for source imaging by division into 80 segments, each containing 7.5 seconds of data of relatively uniform brain activity. For each of these data segments, signals from neuronal sources were isolated using an ICA spatiotemporal decomposition technique designed to extract signals from distinct compact sources that exhibit burst behavior and minimal temporal overlap with other active sources. These ICA signal components have MEG spatial magnetic field patterns corresponding to one or a few spatially distinct compact sources which are much easier to image accurately using the MR-FOCUSS source imaging technique [34]. Separate from the imaging algorithm, the cross-spectrum between ICA signals was calculated. In these cross-spectrum calculations, a sequence of Fast Fourier Transform (FFT) spectra was calculated using $0.5 \mathrm{sec}$ windows with 25\% overlap for 24 frequency bins of $2 \mathrm{~Hz}$ width between 3 and $50 \mathrm{~Hz}$. The imaging results and the signal cross-spectrum were used to calculate the coherence between each voxel/dipole and all other active cortical locations within each of the 24 frequency bins. Finally, for each active source, the average coherence across frequencies and sources was calculated. In these coherence imaging results, the localization of imaged brain activity is strongly dependent on the frequency bands with greatest power.

The axial MRI images with the MEG coherence frequency averages ( $3-50 \mathrm{~Hz}$ ) of the resting-state from all subjects were visually inspected. MEG-Tools identified regions of maximum coherence in individual subjects. Regions of interest investigated in this study are involved in language, memory, decision making, empathy, executive and higher cognitive functioning and correlated to Brodmann's Areas (BA) 9, 10, 24, 46 and 47. These regions have been reported in previous studies to be highly active in patients with schizophrenia [40]. The va- 
riance between the amplitudes of the coherence levels from the patients with schizophrenia and the control subjects were assessed for statistical significance.

\subsection{Statistical Analyses}

Differences between the coherence estimates of the Schizophrenia group and the Control group were assessed using a three-way analysis of variance (ANOVA) with GROUP as the between-group effect, and Brodmann Area region $(9,10,24,46,47)$ and SIDE (hemisphere: left, right) as within-group effects. Upon significant main effects or their interactions, post-hoc analyses were performed using two-tailed t-tests for independent samples to compare coherence values between groups. Correction for multiple correlations was performed using Bonferroni's method. Statistical analyses were performed in IBM SPSS Statistics Package v.19 (IBM Corporation, Armonk, NY, USA).

\section{Results}

The mean coherence across all 24 frequency bins (within the 3 - $50 \mathrm{~Hz}$ band) were calculated and sites of high coherence (red) were co-registered on the MR images. Table 1 describes the mean coherence amplitudes for each of the Brodmann areas investigated. with 95\% confidence interval, and significance values (t-test results, p-uncorrected) for schizophrenia and control subjects. Figure 1 is a graphical representation of these mean coherence amplitudes. Figure 2 depicts the MEG-CSI results for one Patient with schizophrenia and one Control Subject.

\section{Control Brodmann's Area (C-BA) vs. Schizophrenia Brodmann’s Area (S-BA)}

Figure 1 represents a visual display of the mean coherence amplitudes and Table 1 shows a list of all the parameters. A three-way ANOVA with GROUP as the between-group effect, and BA region (10, 9, 24, 46, 47) and

\section{Table 1. Schizophrenia (S) vs. control (C) comparisons.}

\begin{tabular}{|c|c|c|c|c|}
\hline Location & $\mathrm{N}$ & $95 \%$ CI & Mean Coherence & Independent Samples t-Test \\
\hline S-BA9 Left & 12 & 0.113 to 0.203 & 0.158 & \multirow{2}{*}{$\mathrm{t}(22)=2.66, \mathrm{p}=0.001$} \\
\hline C-BA9 Left & 12 & 0.054 to 0.085 & 0.069 & \\
\hline S-BA9 Right & 12 & 0.121 to 0.228 & 0.174 & \multirow{2}{*}{$\mathrm{t}(22)=2.62, \mathrm{p}=0.016$} \\
\hline C-BA9 Right & 12 & 0.059 to 0.123 & 0.091 & \\
\hline S-BA10 Left & 12 & 0.126 to 0.232 & 0.179 & \multirow{2}{*}{$\mathrm{t}(22)=2.89, \mathrm{p}=0.009$} \\
\hline C-BA10 Left & 12 & 0.046 to 0.121 & 0.083 & \\
\hline S-BA10 Right & 12 & 0.134 to 0.226 & 0.180 & \multirow{2}{*}{$\mathrm{t}(22)=3.12, \mathrm{p}=0.005$} \\
\hline C-BA10 Right & 12 & 0.051 to 0.123 & 0.087 & \\
\hline S-BA24 Left & 12 & -0.00025 to 0.01170 & 0.00572 & \multirow{2}{*}{$\mathrm{t}(22)=1.35, \mathrm{p}=0.191$} \\
\hline C-BA24 Left & 12 & -0.00007 to 0.00302 & 0.00148 & \\
\hline S-BA24 Right & 12 & 0.00177 to 0.01652 & 0.00914 & \multirow{2}{*}{$\mathrm{t}(22)=2.38, \mathrm{p}=0.026$} \\
\hline C-BA24 Right & 12 & -0.00014 to 0.00044 & 0.00015 & \\
\hline S-BA46 Left & 12 & 0.125 to 0.245 & 0.185 & \multirow{2}{*}{$\mathrm{t}(22)=1.65, \mathrm{p}=0.113$} \\
\hline C-BA46 Left & 12 & 0.079 to 0.166 & 0.122 & \\
\hline S-BA46 Right & 12 & 0.149 to 0.262 & 0.205 & \multirow{2}{*}{$\mathrm{t}(22)=2.94, \mathrm{p}=0.008$} \\
\hline C-BA46 Right & 12 & 0.065 to 0.142 & 0.103 & \\
\hline S-BA47 Left & 12 & 0.170 to 0.290 & 0.230 & \multirow{2}{*}{$\mathrm{t}(22)=2.45, \mathrm{p}=0.023$} \\
\hline C-BA47 Left & 12 & 0.079 to 0.183 & 0.131 & \\
\hline S-BA47 Right & 12 & 0.163 to 0.269 & 0.216 & \multirow{2}{*}{$\mathrm{t}(22)=3.81, \mathrm{p}=0.001$} \\
\hline C-BA47 Right & 12 & 0.063 to 0.127 & 0.095 & \\
\hline
\end{tabular}




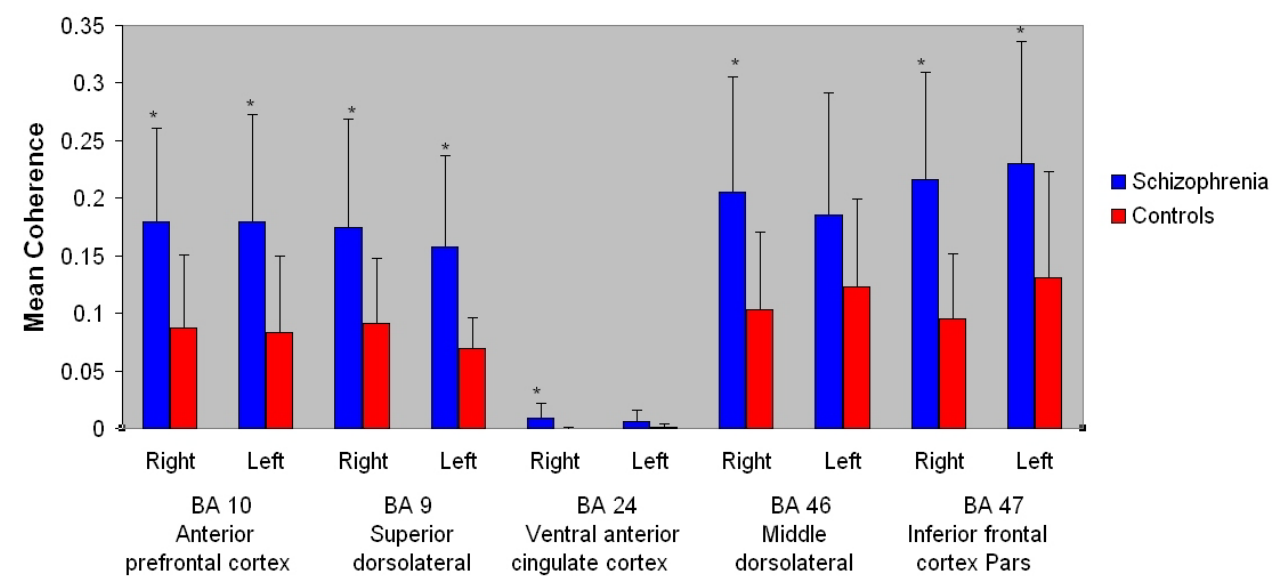

Figure 1. Graph comparing the Mean coherence amplitudes and standard deviations for each of the Brodmann Areas investigated.
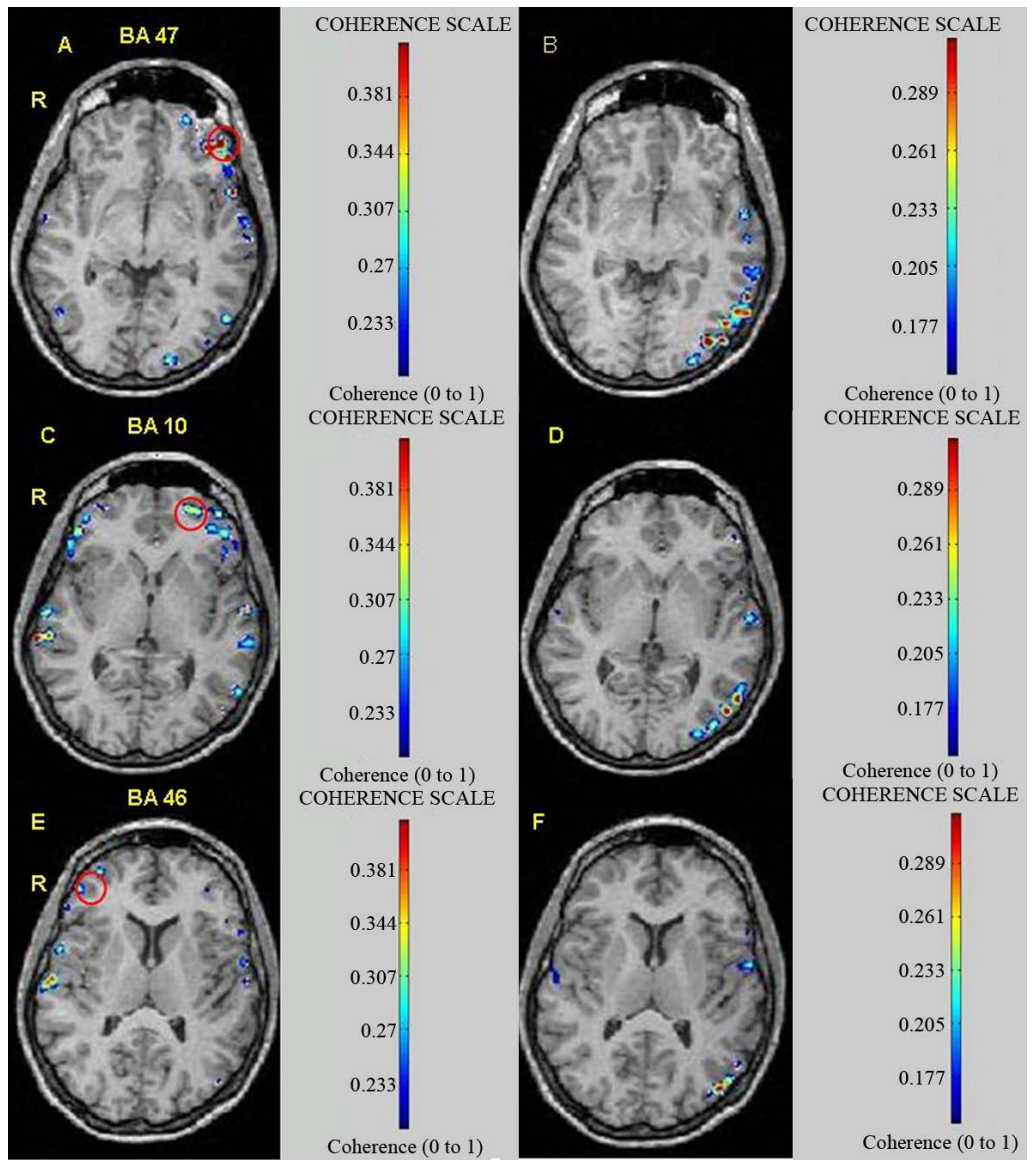

Figure 2. Axial MRI slices with MEG-CSI results overlaid. Left side is one schizophrenic patient on the right side is an age-matched control subject. (A) Brodmann's area 47 is circled indicating this is an area of high coherence; (B) The corresponding results from a Control subject indicating that high coherent areas are only seen in visual association areas (BA 19, 21, 37); (C) Brodmann's area 10 is circled indicating this is an area of high coherence; (D) Control subject coherent areas are only seen in visual association areas (BA 19, 21, 37); (E) Brodmann's area 46 is circled indicating this is an area of high coherence; $(\mathrm{F})$ Control subject coherent areas are only seen in visual association areas (BA 19). Note visual cortical areas are active as the Patient has eyes open. Coherence level is below 0.3 in control subject and greater than 0.3 in schizophrenia patient. 
SIDE (hemisphere: left, right) as within-group effects, showed a significant effect of GROUP $(F(1,22)=12.18$, $\mathrm{p}=0.002)$ and BA region $(\mathrm{F}(1,22)=47.01, \mathrm{p}<0.001)$. A significant BA-by-GROUP $(\mathrm{F}(1,22)=4.45, \mathrm{p}=0.003)$ interaction and a trend towards significance for BA-by-SIDE interaction $(F(1,22)=2.43, p=0.054)$ were also observed. There were no significant effects of $\operatorname{SIDE}(F(1,22)=0.005$, $p=0.94)$, no significant SIDE-byGROUP $(\mathrm{F}(1,22)=0.78, \mathrm{p}=0.38)$ and BA-by-SIDE-by-GROUP $(\mathrm{F}(1,22)=2.42, \mathrm{p}=0.13)$ interactions. Post-hoc two-tailed t-tests for independent samples showed significant differences in coherence values between schizophrenia patients and healthy controls in respective BA regions listed below.

Coherence in the right hemisphere inferior frontal gyrus (BA 47-pars orbitalis), showed a highly significant difference between groups ( $\mathrm{p}<0.01$, corrected). The comparison of the coherence in the left hemisphere BA 47 showed no significant differences after correction of $\mathrm{p}$-value for multiple comparisons ( $\mathrm{p}<0.2$, corrected). The MEG results of BA 47, for one patient and subject, are shown in Figure 2 (top section). The comparison for the right hemisphere superior frontal gyrus (BA 9-dorsolateral prefrontal cortex), also showed no significant differences after correction of $p$-value for multiple comparisons ( $p<0.15$, corrected). The comparison of the left hemisphere coherences for the same region (BA 9) on the other hand showed a significant difference between groups ( $\mathrm{p}<0.01$, corrected).

The comparison of coherence estimates for the left hemisphere middle frontal gyrus (BA 10 - anterior prefrontal cortex) showed a weak trend towards significance after correction for multiple comparisons ( $\mathrm{p}<0.09$, corrected) between control subjects and the patients with schizophrenia. The comparison of the right hemisphere BA 10 values on the other hand showed a significant difference ( $p<0.05$, corrected). MEG results for this area are shown in Figure 2 (middle section).

The comparison of the coherences for right hemisphere middle frontal gyrus (BA 46) (which is also the dorsolateral prefrontal cortex like BA 9) showed a weak trend towards significance after correction for multiple comparisons ( $p$ 0.08). MEG results for BA 46 are shown in Figure 2 (bottom section). The comparison of the left hemisphere BA 46 was not statistically significant ( $p>0.05)$.

The comparison of right hemisphere cingulate gyrus (BA 24-ventral anterior cingulate cortex) showed a significant difference between group ( $\mathrm{p}<0.05$, uncorrected). The comparison of the left hemisphere BA 10 values on the other hand showed no significant differences between groups ( $p>0.05)$.

\section{Discussion}

These preliminay investigations show that areas of the frontal pole cortex (BA 10), dorsolateral prefrontal cortex (BA 9 and 46), orbitofrontal cortex (BA 10 and 47), and the anterior cingulate cortexes (BA 24) in patients with schizophrenia exhibit noticeably higher coherence levels in a resting state compared to controls. BA 47 in the ventral-orbital frontal cortex has been largely associated with multitasking and has been implicated in the processing of syntax in spoken and sign languages, and more recently in musical [41]. Current research suggests that BA 10 in the frontopolar part of the frontal cortex is involved in strategic processes in memory retrieval and executive function [42] [43]. BA 9 (which is the dorsolateral prefrontal cortex, the highest cortical area responsible for motor planning, organization, and regulation) plays an important role in the integration of sensory and mnemonic information and the regulation of intellectual function and action, and is also involved in working memory. BA 46 (which is also located in the dorsolateral prefrontal cortex like BA 9) is involved similarly in the higher cortical functioning. BA 24 (the anterior cingulate cortex) is responsible for rational cognitive functions, such as reward anticipation, decision-making, and empathy [44]-[48]. The mentioned functional significances of these areas may indicate that patients with schizophrenia have an over-recruitment of activity in the frontal lobe of the brain, even during resting-state. These data add to the findings by Sperling et al., [49] which showed that a group of patients with schizophrenia differed overall from the healthy subjects in the elevation of absolute dipole values measured in both hemispheres. For the region of slow dipole activity (2 - $6 \mathrm{~Hz}$ ), a high correlation was found between the intensity of dipole concentration and productive psychotic symptoms (PANSS, P1-P7). Dipole localization (for both frequency ranges) showed a concentration effect (DCE) in the temporoparietal region in patients with schizophrenia.

A recent study using MEG has found similar activation of the frontal polar cortex, and prefrontal cortex [40]. In the current study, we find additional activation in the anterior cingulate region (BA 24) in schizophrenia patients when compared to controls directly. BA 24 is responsible for rational cognitive functions, such as reward anticipation, decision-making, and empathy. Therefore, coherent activity seen in patients with schizophrenia in 
this area may imply that it is actively being inhibited by other regions possibly leading to decreased display of empathy or rational decision making. It could also mean that the area is constantly engaged in other tasks and therefore unable to provide needed activation to have empathy or the ability to make rational decisions.

A second MEG resting-state study examined gamma frequency band in patients with schizophrenia and controls and similar to the findings of the current paper, detected activation in the posterior region of the brain in both controls and schizophrenia patients [33]. The findings of activation in the occipital region, was also reported in another MEG study [50], however, they found no definite coherence increases in various areas of the frontal lobe such as we found in the current study. Our data emphasized the high coherence in many areas of the frontal lobe (BA 9, 10, 46, 47), and undersores the significant deviations in this region in subjects with schizophrenia. Further speculation on the possible implications of the limited pilot data provided here must await independent replication in a larger sample.

The control data matched the results of a previous MEG study [37]. Generally, no substantial coherence was detected in any other area but the occipital lobe. This correlated well with visual activation since as the subjects' eyes were open during the entirety of both of these studies.

Our MEG findings can be used in the future to detect possible biomarkers/indicators of an abnormal resting-state in schizophrenia patients and could possibly help further define the clinical subtypes of the disorder. As no task performance or cooperativeness is required (other than staying still during recording), this procedure may be useful in uncooperative or significantly cognitively imparied patients. Furthermore, DMN indicators recorded by MEG can be used to quantitatively assess brain activation levels pre- and post-treatments in patients with schizophrenia. DMN analysis with MEG can also evaluate various treatments effectiveness, whether the treatment is via drugs, transcranial magnetic stimulation, deep brain stimulation, or other therapies, thus providing a valuable clinical tool in the mental health field to evaluate the patients progress.

Though there is continuous activation of regions in the frontal lobe in patients with schizophrenia, it remains to be seen where other areas of the brain may be aroused during the experience of certain active symptoms of the disease: auditory hallucinations, visual hallucinations, etc.

Three major drawbacks limit the generalizability of the results. First is the small sample size. Definitive correlations with clinical symptom-clusters could not be provided. Furthermore, for the purposes of this preliminary exploration subjects were not matched for gender. While early work suggested that there may be gender-based differences in the clinical symptomatology [51], evidence for gender-based electrophysiological differences within schizophrenia populations has been sparse [52]. Nonetheless, this issue needs to be documented in subsequent studies utilizing coherence source imaging with larger sample sizes allowing the examination of any possible gender-based differences. Secondly, patients were medicated with various antipsychotic agents (majority on atypical antipsychotics). The effects of medications on the MEG signal are currently not well-known. Furthermore, as in coherence imaging results, the localization of imaged brain activity is strongly dependent on the frequency bands with greatest power, investigation of coherent activity at narrow preselected bands is warranted.

\section{References}

[1] Kurt, H., Dikmen, M., Basaran, A., Yenilmez, C., Ozdemir, F., Degirmenci, I., Gunes, H.V., Kucuk, M.U. and Mutlu, F. (2011) Dopamine D2 Receptor Gene-141C Insertion/Deletion Polymorphism in Turkish Schizophrenic Patients. Molecular Biology Reports, 38, 1407-1411. http://dx.doi.org/10.1007/s11033-010-0244-6

[2] Barrett, E.A., Sundet, K., Faerden, A., Agartz, I., Bratlien, U., Romm, K.L., Mork, E., Rosberg, J.I., Steen, N.E., Andreassen, O.A. and Melle, I. (2010) Suicidality in First Episode Psychosis Is Associated with Insight and Negative Beliefs about Psychosis. Schizophrenia Research, 123, 257-262. http://dx.doi.org/10.1016/j.schres.2010.07.018

[3] Jepsen, J.R., Fagerlund, B., Pagsberg, A.K., Christensen, A.M., Nordentoft, M. and Mortensen, E.L. (2010) Deficient Maturation of Aspects of Attention and Executive Functions in Early Onset Schizophrenia. European Child \& Adolescent Psychiatry, 19, 773-786. http://dx.doi.org/10.1007/s00787-010-0126-4

[4] Voss, M., Moore, J., Hauser, M., Gallinat, J., Heinz, A. and Haggard, P. (2010) Altered Awareness of Action in Schizophrenia: A Specific Deficit in Predicting Action Consequences. Brain, 133, 3104-3112. http://dx.doi.org/10.1093/brain/awq152

[5] Keshavan, M.S., Nasrallah, H.A. and Tandon, R. (2011) Schizophrenia, “Just the Facts” 6. Moving ahead with the Schizophrenia Concept: From the Elephant to the Mouse. Schizophrenia Research, 127, 3-13. http://dx.doi.org/10.1016/j.schres.2011.01.011 
[6] Qi, Z., Miller, G. and Voit, E.O. (2010) The Neuregulin Signaling Pathway and Schizophrenia: From Genes to Synapses and Neural Circuits. Pharmacopsychiatry, 43, S50-S60. http://dx.doi.org/10.1055/s-0030-1248317

[7] Hoffman, E.J. and State, M.W. (2010) Progress in Cytogenetics: Implications for Child Psychopathology. Journal of the American Academy of Child \& Adolescent Psychiatry, 49, 736-751. http://dx.doi.org/10.1016/j.jaac.2010.03.016

[8] Murray, R.M., Lappin, J. and Di Forti, M. (2008) Schizophrenia: From Developmental Deviance to Dopamine Dysregulation. European Neuropsychopharmacology, 18, S129-S134. http://dx.doi.org/10.1016/j.euroneuro.2008.04.002

[9] Javitt, D.C. (2007) Glutamate and Schizophrenia: Phencyclidine, N-Methyl-D-Aspartate Receptors, and DopamineGlutamate Interactions. International Review Neurobiology, 78, 69-108. http://dx.doi.org/10.1016/S0074-7742(06)78003-5

[10] Ascher-Svanum, H., Zhu, B., Faries, D.E., Salkever, D., Slade, E.P., Peng, X. and Conley, R.R. (2010) The Cost of Relapse and the Predictors of Relapse in the Treatment of Schizophrenia. BMC Psychiatry, 10, 1-7. http://dx.doi.org/10.1186/1471-244X-10-2

[11] Knyazeva, M.G., Jalili, M., Meuli, R., Hasler, M., De Foe, O. and Do, K.Q. (2008) Alpha Rhythm and Hypofrontality in Schizophrenia. Acta Psychiatrica Scandinavica, 118, 188-199. http://dx.doi.org/10.1111/j.1600-0447.2008.01227.x

[12] Harris, A.W., Bahramali, H., Slewa-Younan, S., Gordon, E., Williams, L. and Li, W.M. (2001) The Topography of Quantified Electroencephalography in Three Syndromes of Schizophrenia. International Journal of Neuroscience, 107, 265-278. http://dx.doi.org/10.3109/00207450109150689

[13] Boutros, N.N., Arfken, C., Galderisi, S., Warrick, J., Pratt, G. and Iacono, W. (2008) The Status of EEG Abnormality as a Diagnostic Test for Schizophrenia. Schizophrenia Research, 99, 225-237. http://dx.doi.org/10.1016/j.schres.2007.11.020

[14] Jalili, M., Lavoie, S., Deppen, P., Meuli, R., Do, K.Q., Cuenod, M., Hasler, M., De Feo, O. and Knyazeva, M.G. (2007) Dysconnection Topography in Schizophrenia Revealed with State-Space Analysis of EEG. PLOS One, 2, e1059. http://dx.doi.org/10.1371/journal.pone.0001059

[15] Raichle, M.E., MacLeod, M.A., Snyder, A.Z., Powers, W.J., Gusnard, D.A. and Shulman, G.L. (2001) A Default Mode of Brain Function. Proceedings of the National Academy of Sciences of the USA, 98, 676-682. http://dx.doi.org/10.1073/pnas.98.2.676

[16] Laufs, H. (2008) Endogenous Brain Oscillations and Related Networks Detected by Surface EEG-Combined fMRI. Human Brain Mapping, 29, 762-769. http://dx.doi.org/10.1002/hbm.20600

[17] Fransson, P. (2006) How Default Is the Default Mode of Brain Function? Further Evidence from Intrinsic BOLD Signal Fluctuations. Neuropsychologia, 44, 2839-2845. http://dx.doi.org/10.1016/j.neuropsychologia.2006.06.017

[18] Thomason, M.E., Chang, C.E., Glover, G.H., Gabrieli, J.D., Greicius, M.D. and Gotlib, I.H. (2008) Default-Mode Function and Task-Induced Deactivation Have Overlapping Brain Substrates in Children. NeuroImage, 41, 1493-1503. http://dx.doi.org/10.1016/j.neuroimage.2008.03.029

[19] Pyka, M., Beckmann, C.F., Schöning, S., Hauke, S., Heider, D., Kugel, H., Arolt, V. and Konrad, C. (2009) Impact of Working Memory Load on fMRI Resting State Pattern in Subsequent Resting Phases. PLOS One, 4, e7198. http://dx.doi.org/10.1371/journal.pone.0007198

[20] Fox, M.D., Zhang, D., Snyder, A.Z. and Raichle, M.E. (2009) The Global Signal and Observed Anticorrelated Resting State Brain Networks. Journal Neurophysiology, 101, 3270-3283. http://dx.doi.org/10.1152/jn.90777.2008

[21] Ongür, D., Lundy, M., Greenhouse, I., Shinn, A.K., Menon, V., Cohen, B.M. and Renshaw, P.F. (2010) Default Mode Network Abnormalities in Bipolar Disorder and Schizophrenia. Psychiatry Research, 183, 59-68. http://dx.doi.org/10.1016/j.pscychresns.2010.04.008

[22] Tu, P.C., Lee, Y.C., Chen, Y.S., Li, C.T. and Su, T.P. (2013) Schizophrenia and the Brain's Control Network: Aberrant within- and between-Network Connectivity of the Frontoparietal Network in Schizophrenia. Schizophrenia Research, 147, 339-347. http://dx.doi.org/10.1016/j.schres.2013.04.011

[23] Salmelin, R. and Kujala, J. (2006) Neural Representation of Language: Activation versus Long-Range Connectivity. Trends in Cognitive Sciences, 10, 519-525. http://dx.doi.org/10.1016/j.tics.2006.09.007

[24] Hamalainen, M., Hari, R., Ilmoniemi, J., Knuutila, J. and Lounamaa, O.V. (1993) Magnetoencephalography-Theory, Instrumentation and Applications to Noninvasive Studies of the Working Human Brain. Review of Modern Physics, 65, 413-497. http://dx.doi.org/10.1103/RevModPhys.65.413

[25] Morika, T., Hayashib, M. and Hori, T. (1997) Auto Power and Coherence Analysis of Delta-Theta Band EEG during Waking-Sleeping Transition Period. Electroencephalography and Clinical Neurophysiology, 103, 633-641. http://dx.doi.org/10.1016/S0013-4694(97)00048-5

[26] Georgopoulos, A.P., Karageorgiou, E., Leuthold, A.C., Lewis, S.M., Lynch, J.K., Alonso, A.A., Aslam, Z., Carpenter, A.F., Georgopoulos, A., Hemmy, L.S., Koutlas, I.G., Langheim, F.J.P., McCarten, J.R., McPherson, S.E., Pardo, J.V., 
Pardo, P.J., Parry, G.J., Rottunda, S.J., Segal, B.M., Sponheim, S.R., Stanwyck, J.J., Stephane, M. and Westermeyer, J.J. (2007) Synchronous Neural Interactions Assessed by Magnetoencephalography: A Functional Biomarker for Brain Disorders. Journal of Neural Engineering, 4, 349-355. http://dx.doi.org/10.1088/1741-2560/4/4/001

[27] Fehr, T., Kissler, J., Wienbruch, C., Moratti, S., Elbert, T., Watzl, H. and Rockstroh, B. (2003) Source Distribution of Neuromagnetic Slow-Wave Activity in Schizophrenic Patients-Effects of Activation. Schizophrenia Research, 63, 63-71. http://dx.doi.org/10.1016/S0920-9964(02)00213-X

[28] Cañive, J.M., Lewine, J.D., Edgar, J.C., Davis, J.T., Miller, G.A., Torres, F. and Tuason, V.B. (1998) Spontaneous Brain Magnetic Activity in Schizophrenia Patients Treated with Aripiprazole. Psychopharmacology Bulletin, 34, 101105.

[29] Sperling, W., Vieth, J., Martus, M., Demling, J. and Barocka, A. (1999) Spontaneous Slow and Fast MEG Activity in Male Schizophrenics Treated with Clozapine. Psychopharmacology, 142, 375-382. http://dx.doi.org/10.1007/s002130050902

[30] Belardinelli, P., Ciancetta, L., Staudt, M., Pizzella, V., Londei, A., Birbaumer, N., Romani, G.L. and Braun, C. (2007) Cerebro-Muscular and Cerebro-Cerebral Coherence in Patients with pre- and Perinatally Acquired Unilateral Brain Lesions. NeuroImage, 37, 1301-1314. http://dx.doi.org/10.1016/j.neuroimage.2007.05.053

[31] Hinkley, L.B., Vinogradov, S., Guggisberg, A.G., Fisher, M., Findlay, A.M. and Nagarajan, S.S. (2011) Clinical Symptoms and Alpha Band Resting-State Functional Connectivity Imaging in Patients with Schizophrenia: Implications for Novel Approaches to Treatment. Biological Psychiatry, 70, 1134-1142. http://dx.doi.org/10.1016/j.biopsych.2011.06.029

[32] Uhlhaas, P.J. and Singer, W. (2010) Abnormal Neural Oscillations and Synchrony in Schizophrenia. Natural Reviews Neuroscience, 11, 100-113. http://dx.doi.org/10.1038/nrn2774

[33] Rutter, L., Carver, F.W., Holroyd, T., Nadar, S.R., Mitchell-Francis, J., Apud, J., Weinberger, D.R. and Coppola, R. (2009) Magnetoencephalographic Gamma Power Reduction in Patients with Schizophrenia during Resting Condition. Human Brain Mapping, 30, 3254-3264. http://dx.doi.org/10.1002/hbm.20746

[34] Moran, J.E., Bowyer, S. and Tepley, N. (2005) Multi-Resolution FOCUSS: A Source Imaging Technique Applied to MEG Data. Brain Topography, 18, 1-17. http://dx.doi.org/10.1007/s10548-005-7896-X

[35] Moran, J.E., Drake, C.L. and Tepley, N. (2004) ICA Methods for MEG Imaging. Neurophysiology and Clinical Neurophysiology, 72.

[36] Elisevich, K., Shukla, N., Moran, J.E., Smith, B., Schultz, L., Mason, K., Barkley, G.L., Tepley, N., Gumenyuk, V. and Bowyer, S.M. (2011) An Assessment of MEG Coherence Imaging in the Study of Temporal Lobe Epilepsy. Epilepsia, 52, 1110-1119. http://dx.doi.org/10.1111/j.1528-1167.2011.02990.x

[37] de Pasquale, F., Penna, S.D., Snyder, A.Z., Lewis, C., Mantini, D., Marzetti, L., Belardinelli, P., Ciancetta, L., Pizzella, V. and Romani, G.L. (2010) Temporal Dynamics of Spontaneous MEG Activity in Brian Networks. Proceedings of the National Academy of Sciences of the United States of America, 107, 6040-6045. http://dx.doi.org/10.1073/pnas.0913863107

[38] Bowyer, S.M., Mason, K., Tepley, N., Smith, B. and Barkley, G.L. (2003) Magnetoencephalographic Validation Parameters for Clinical Evaluation of Interictal Epileptic Activity. Journal of Clinical Neurophysiology, 20, 87-93. http://dx.doi.org/10.1097/00004691-200304000-00001

[39] Moran, J. (2004) MEG Tools Software Program. http://www.megimaging.com

[40] Kim, J.S., Shin, K.S., Jung, W.H., Kim, S.N., Kwon, J.S. and Chung, C.K. (2014) Power Spectral Aspects of the Default Mode Network in Schizophrenia: An MEG Study. BMC Neuroscience, 15, 104. http://dx.doi.org/10.1186/1471-2202-15-104

[41] Menon, V. and Levitin, D. (2005) The Rewards of Music Listening: Response and Physiological Connectivity of the Mesolimbic System. NeuroImage, 28, 175-184. http://dx.doi.org/10.1016/j.neuroimage.2005.05.053

[42] Ongür, D., Ferry, A.T. and Price, J.L. (2003) Architectonic Subdivision of the Human Orbital and Medial Prefrontal Cortex. Journal of Comparative Neurology, 460, 425-449. http://dx.doi.org/10.1002/cne.10609

[43] Ramnani, N. and Owen, A.M. (2004) Anterior Prefrontal Cortex: Insights into Function from Anatomy and Neuroimaging. Nature Reviews Neuroscience, 5, 184-194. http://dx.doi.org/10.1038/nrn1343

[44] Medford, N. and Critchley, H.D. (2010) Conjoint Activity of Anterior Insular and Anterior Cingulate Cortex: Awareness and Response. Brain Structure and Function, 214, 535-549. http://dx.doi.org/10.1007/s00429-010-0265-X

[45] Raine, A. and Yang, Y. (2006) Neural Foundations to Moral Reasoning and Antisocial Behavior. Social Cognitive \& Affective Neurosci, 1, 203-213. http://dx.doi.org/10.1093/scan/nsl033

[46] Peters, J. and Büchel, C. (2010) Neural Representations of Subjective Reward Value. Behavioural Brain Research, 213, 135-141. http://dx.doi.org/10.1016/j.bbr.2010.04.031 
[47] Shoeunbaum, G. and Esber, G.R. (2010) How Do You (Estimate You Will) Like Them Apples? Integration as Defining Trait of Orbitofrontal Function. Current Opinion in Neurobiology, 20, 205-211. http://dx.doi.org/10.1016/j.conb.2010.01.009

[48] Rosen, H.R. and Rich, B.A. (2010) Neurocognitive Correlates of Emotional Stimulus Processing in Pediatric Bipolar Disorder: A Review. Postgraduate Medicine, 122, 94-104. http://dx.doi.org/10.3810/pgm.2010.07.2177

[49] Sperling, W., Martus, P., Kober, H., Bleich, S. and Kornhuber, J. (2002) Spontaneous, Slow and Fast Magnetoencephalographic Activity in Patients with Schizophrenia. Schizophrenia Research, 58, 189-199. http://dx.doi.org/10.1016/S0920-9964(02)00238-4

[50] Mannell, M.V., Franco, A.R., Calhoun, V.D., Cañive, J.M., Thoma, R.J. and Mayer, A.R. (2010) Resting State and Task-Induced Deactivation: A Methodological Comparison in Patients with Schizophrenia and Healthy Controls. Human Brain Mapping, 31, 424-437.

[51] Flor-Henry, P. (1990) Influence of Gender in Schizophrenia as Related to Other Psychopathological Syndromes. Schizophrenia Bulletin, 16, 211-227. http://dx.doi.org/10.1093/schbul/16.2.211

[52] Slewa-Younan, S., Gordon, E., Harris, A.W., Haig, A.R., Brown, K.J., Flor-Henry, P. and Williams, L.M. (2004) Sex Differences in Functional Connectivity in First-Episode and Chronic Schizophrenia Patients. American Journal of Psychiatry, 161, 1595-1602. http://dx.doi.org/10.1176/appi.ajp.161.9.1595 
Scientific Research Publishing (SCIRP) is one of the largest Open Access journal publishers. It is currently publishing more than 200 open access, online, peer-reviewed journals covering a wide range of academic disciplines. SCIRP serves the worldwide academic communities and contributes to the progress and application of science with its publication.

Other selected journals from SCIRP are listed as below. Submit your manuscript to us via either submit@scirp.org or Online Submission Portal.
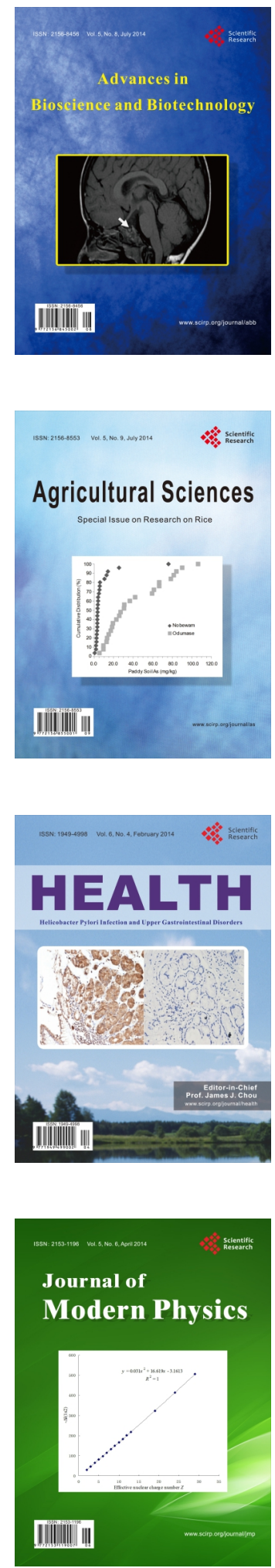
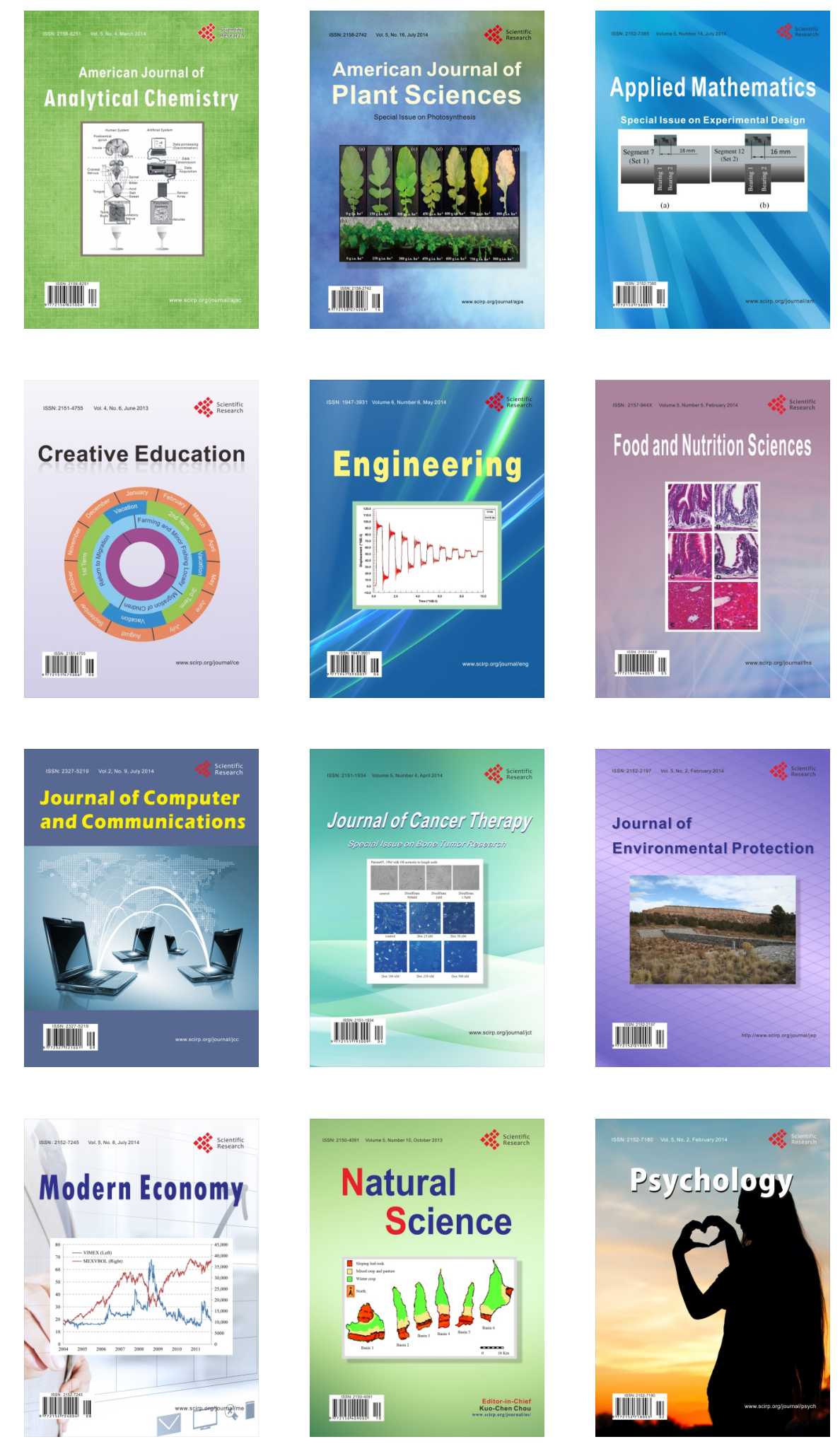